\title{
Tacrolimus-associated thrombotic microangiopathy in a lung transplant recipient
}

\author{
Nathan Lewis Boyer, ${ }^{1}$ Alexander Niven, ${ }^{1}$ Jeffery Edelman ${ }^{2}$
}

${ }^{1}$ Department of Internal Medicine, Madigan Army Medical Center, Tacoma, Washington, USA ${ }^{2}$ Pulmonary/Critical Care Division, University of Washington, Seattle, Washington, USA

Correspondence to Dr Nathan Lewis Boyer, nathan.boyer1@gmail.com
To cite: Boyer NL, Niven A, Edelman J. BMJ Case Rep Published online: [please include Day Month Year] doi:10.1136/bcr-2012007351

\begin{abstract}
SUMMARY
A 25-year-old woman with a history of bilateral lung transplant secondary to cystic fibrosis presented with non-specific abdominal complaints and was found to have acute kidney injury, thrombocytopaenia and laboratory findings consistent with a microangiopathic haemolytic anaemia. Her thrombotic microangiopathy (TMA) was attributed to tacrolimus, which was discontinued and replaced with cyclosporine with resolution of her TMA and no subsequent complications. This is the fifth reported case of TMA associated with tacrolimus use in a lung transplant patient, and the third to be successfully managed with cyclosporine substitution. Clinicians must be aware of this uncommon, but likely under-reported complication of tacrolimus therapy in lung transplant recipients. Cyclosporine replacement may be used as a successful therapy to treat tacrolimus-associated TMA without increasing the risk of acute rejection that may be associated with other treatment strategies.
\end{abstract}

\section{BACKGROUND}

As the number of transplant recipients and their survival increase, awareness of common and complex management issues associated with lung transplantation is increasingly important for both primary care and subspecialty providers. Transplant-associated thrombotic microangiopathy (TA-TMA) is an increasingly well-recognised complication in these patients, and is similar but clinically distinct from other thrombotic microangiopathies like thrombotic thrombocytopaenic purpura (TTP). ${ }^{1}$ Calcineurin inhibitors such as tacrolimus are commonly implicated in the development of TA-TMA after both solid organ and haematopoietic stem cell transplantation, which can occur at any time after initiation of therapy. The disorder rarely presents with the classic pentad of symptoms, and can be fatal if left undiagnosed. ${ }^{1-3}$ There are scant case reports of TA-TMA in lung transplant recipients, creating significant potential that this serious disease may be missed outside specialty centres. Early discontinuation of the offending agent may be the most promising treatment strategy for this condition, with additional adjunctive therapies including therapeutic plasma exchange (TPE) reserved for more serious or refractory cases. Replacement of tacrolimus with cyclosporine may represent an effective, low risk management strategy of TA-TMA that reduces the risk of acute rejection complications. ${ }^{14}$

\section{CASE PRESENTATION}

A 25 -year-old woman with medical history of bilateral lung transplant 15 months earlier for complications of cystic fibrosis presented to the emergency department with a week of severe, progressive fatigue, easy bruising, decreased appetite, nausea, abdominal pain and a single episode of vomiting. Her medical history included two prior episodes of acute rejection, a history of successfully treated childhood acute lymphocytic leukaemia and chronic active hepatitis $\mathrm{C}$ with a recent viral count of $4550000 \mathrm{IU} / \mathrm{ml}$; prior liver biopsy demonstrated grade 1 inflammation and stage 0 fibrosis. Her immunosuppressive regimen included tacrolimus $4.5 \mathrm{mg}$ in the morning and $4 \mathrm{mg}$ at night, prednisone $10 \mathrm{mg}$ daily, mycophenylate $720 \mathrm{mg}$ twice a day and sirolimus $3 \mathrm{mg}$ twice a day with prophylactic trimethoprim/sulfamethoxazole. She is married and denied HIV risk factors. Physical examination was significant for multiple ecchymoses and mild diffuse abdominal tenderness without peritoneal signs. She did not have any other localising signs of infection.

\section{INVESTIGATIONS}

Initial laboratory evaluation demonstrated a normocytic anaemia ( $\mathrm{Hgb} 8 \mathrm{~g} / \mathrm{dl}$ ) decreased from her prior baseline of $11.4 \mathrm{~g} / \mathrm{dl} 4$ months earlier, and a platelet count of $54 \times 10(9) / 1(159 \times 10(9) / 110$ days earlier). Creatine was elevated at $3 \mathrm{mg} / \mathrm{dl}$ (baseline $0.84 \mathrm{mg} / \mathrm{dl}$ ) and liver function tests were normal. Evaluation of a peripheral blood smear confirmed her anaemia and thrombocytopaenia and demonstrated a significant number of schistocytes. Lactate dehydrogenase was elevated at 886 units $/ 1$ and haptoglobin was markedly decreased, suggesting haemolysis. Prothrombin and partial thromboplastin times were normal. Acute abdominal series was normal and right upper quadrant ultrasound showed no evidence of gall bladder or biliary abnormalities. Tacrolimus and sirolimus levels were normal at 8 and $5 \mathrm{ng} / \mathrm{ml}$, respectively. $\beta$-HCG was negative, and her ADAMTS13 activity level was $76 \%$ (normal $>60 \%$ ). The Naranjo Score, a commonly recognised tool to assess the likelihood that a change in clinical status is the result of an adverse drug reaction rather than disease progression or other factors, was 5 (adverse drug reaction probable).

\section{DIFFERENTIAL DIAGNOSIS}

TA-TMA belongs to the family of thrombotic microangiopathies (TMAs), which include TTP, haemolytic uremic syndrome, pregnancy-associated microangiopathy and microangiopathic haemolytic anaemia 
(MAHA) associated with a variety of systemic vasculidities and other autoimmune disorders. ${ }^{1356}$ TMA is a syndrome that represents a final common pathway of endothelial injury, microcirculatory thrombosis and fibrin deposition, leading to microangiopathic haemolytic anaemia and platelet consumption.

TTP is classically described as a pentad of fever, neurological and renal impairment, MAHA and thrombocytopaenia without another underlying aetiology. ${ }^{1}{ }^{3}$ Idiopathic TTP is typically due to an inherited defect, genetic mutation or acquisition of inhibitory antibodies to ADAMTS13, a protease enzyme responsible for cleaving large von Willebrand factor (VWF) multimers. Treatment includes plasma infusion or TPE, with other immunosuppressive agents employed with variable success in refractory cases. TA-TMA and TTP were originally thought to be due to similar mechanisms of endothelial damage; however, low ADAMTS13 levels are rarely found in TA-TMA and the benefit of TPE remains uncertain based on the pooled data published in the latest TA-TMA consensus guidelines. ${ }^{1}$

There are multiple potential contributing causes to the development of TA-TMA. The disorder is most often seen in haematopoetic stem cell transplant (HSCT) patients, more commonly in allogeneic than autologous transplant recipients, with a reported incidence of $10-25 \% .^{12}$ The calcineurin inhibitors cyclosporine and tacrolimus have been identified as contributing factors to TA-TMA in both HSCT and solid organ transplant recipients, with endothelial damage from tissue ischaemia at the time of transplantation, an additional risk factor in renal transplant patients. ${ }^{178}$ Use of sirolimus in addition to tacrolimus for graft-versus-host disease (GVHD) prophylaxis has been associated with increased risk of TA-TMA, although these individuals appear to have a more favourable renal prognosis and overall survival compared with TA-TMA patients on a calcineurin agent alone.

\section{TREATMENT}

Our patient received intravenous fluid resuscitation, and tacrolimus, sirolimus and trimethoprim/sulfamethoxazole were discontinued. With this conservative therapy, her acute kidney injury, thrombocytopaenia and laboratory evidence of MAHA rapidly improved (figures 1 and 2). She was discharged on hospital day 4 with prednisone $10 \mathrm{mg}$ daily, mycophenylate $720 \mathrm{mg}$ twice a day, trimethoprim/sulfamethoxazole DS 1 tab daily and was started on cyclosporine $200 \mathrm{mg}$ twice a day.

\section{OUTCOME AND FOLLOW-UP}

The patient demonstrated no clinical evidence of recurrent TMA or short-term evidence of acute rejection upon close

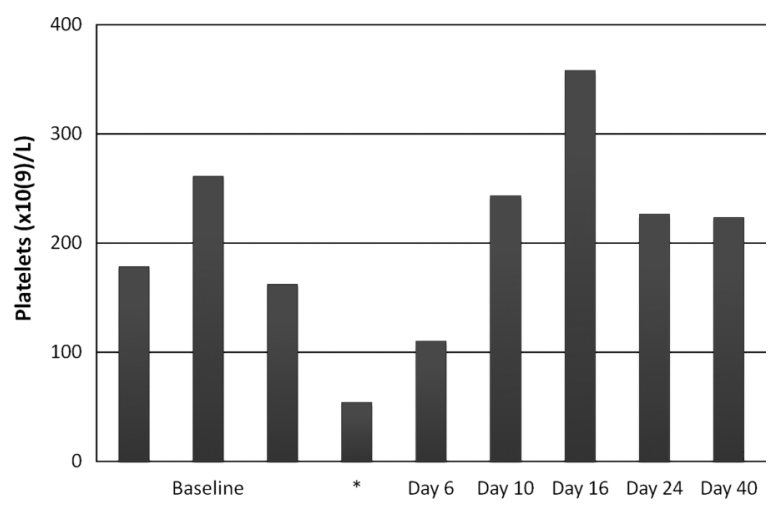

Figure 1 Platelets: baseline over 6 months prior to presentation, * represents the day of presentation and 40 days after presentation. follow-up and re-evaluation. Over the next month, the patient received frequent lab draws that demonstrated complete resolution of her anaemia, thrombocytopaenia, and acute kidney injury to baseline levels. Based on this rapid clinical response, tacrolimus was felt to be the offending agent and she was continued on cyclosporine without rechallenge to her prior immunosuppressive regimen. Although she has experienced further transplant related complications since this event, she has not demonstrated further evidence of TMA over 2.5 subsequent years of serial follow-up.

\section{DISCUSSION}

Tacrolimus, a derivative of the fungus Streptomyces tsukubaensis, is a macrolide lactone and is used as a potent immunosuppressant in organ transplantation. It suppresses cellular immunity by binding to an intracellular protein, FKBP-12, and complexing with calcineurin-dependent proteins to inhibit calcineurin phosphatase activity and T-lymphocyte activation. ${ }^{1} 2788$ TMA, a rare but serious complication secondary to tacrolimus, has been reported in $1-4.7 \%$ of adult transplant patients. In a review of 91 solid organ transplant recipients with tacrolimus-associated TMA, 81\% of cases were seen in renal transplant patients, $8 \%$ in liver transplant patients, and less than $1 \%$ in lung and heart transplant patients. $^{7-10}$ TMA is more common in women and typically presents within the first year of transplant, although the reaction appears to be independent of both the duration and serum levels of tacrolimus. Severity of presentation can range from an asymptomatic elevation in serum creatine to the classic pentad of clinical symptoms, and can be fatal if diagnosis is delayed. The mechanism of tacrolimus-associated TMA is unknown, although postulated mechanisms of endothelial injury include direct cytotoxic damage and injury mediated by platelet activation, elevated VWF and thrombomodulin, altered complement regulation and decreased nitric oxide and prostacyclin production.

Without treatment, TMA is associated with significant mortality. Contrary to the clinical experience with TTP, a summary of 11 heterogeneous clinical studies using TPE for TA-TMA in HSCT recipients demonstrated a median clinical response of only $36.5 \%$ (range $0-80 \%$ ) and an associated mortality of $80 \%$ (range 4 $100 \%){ }^{128}$ In a transplant patient where TMA is believed to be caused by calcineurin inhibitors, removal or dose reduction of the offending agent and supportive care alone have been shown to be an effective treatment strategy, but are not without risk. In one case series of 21 transplant patients with tacrolimus-induced TMA who were treated with drug discontinuation, 2 patients $(10 \%)$

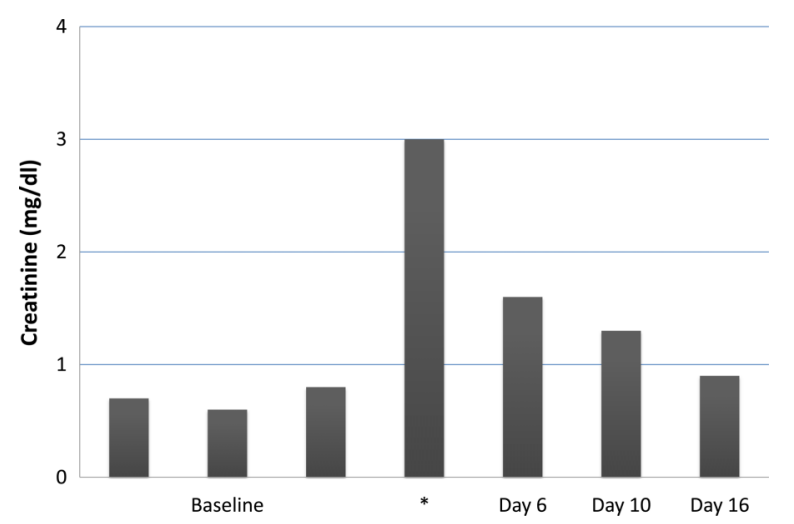

Figure 2 Creatine: baseline over 6 months prior to presentation, * represents the day of presentation and 40 days after presentation. 
subsequently lost their grafts due to acute on chronic rejection and $3(14 \%)$ died of multiple organ failure.

Another published strategy to mitigate the risk of rejection and other complications of reduced immunosuppression is to replace tacrolimus with another immunosuppressive agent, typically cyclosporine. Although cyclosporine is known to lead to TMA in up to $3 \%$ of renal transplant patients, it has been shown to provide effective immunosupression without perpetuating evidence of TMA in cases due to tacrolimus. This is believed to be possible because cyclosporine binds to different cytosolic proteins within target cells as well as having a different molecular structure.

Only three other cases have been published describing tacrolimus-induced TMA in lung transplant recipients. ${ }^{8-10}$ All patients, with the exception of our case, were older (mean age, SD $58 \pm 4$ years old), and had received lung transplantation for severe, lifestyle limiting chronic obstructive pulmonary disease. Including our patient, the majority (3 of 4 ) of patients were women and had developed TMA greater than 12 months after transplant $(8,15,24$ and 30 months), in contrast to commonly associated demographic features in other solid organ transplant populations. In all cases, the offending agent was stopped; cyclosporine was initiated in its place. All patients demonstrated complete recovery from their TMA with therapy. No patients demonstrated evidence of short-term acute rejection as a result of these changes to their immunosuppressive regimen. One patient did develop cyclosporine toxicity and acute kidney injury, which resolved with discontinuation of cyclosporine and conservative management.

Although rare, TA-TMA can be associated with a high death rate unless recognised and treated appropriately. We reported a case of tacrolimus-associated TA-TMA that resolved without GVHD or other complications by stopping the offending agent and then replacing her current immunosuppressive regimen with cyclosporine. High suspicion by primary care providers can help manage these complex medical issues in transplant recipients.

\section{Learning points}

- Transplant thrombotic microangiopathy is a rare but described complication of tacrolimus therapy, which can be life threatening without recognition and appropriate treatment.

- Tacrolimus-induced thrombotic microangiopathy may be more frequent than previously recognised in lung transplant recipients, and can occur later in the post-transplant course than is seen in other solid organ transplant patients.

- Discontinuation of tacrolimus and initiation of cyclosporine therapy can be an effective management strategy in lung transplant patients to treat this condition and prevent short-term complications of acute rejection.
Competing interests None.

Patient consent Obtained.

Provenance and peer review Not commissioned; externally peer reviewed.

\section{REFERENCES}

1 Ho VT, Cutler C, Carter S, et al. Blood and marrow transplant clinical trials network toxicity committee consensus summary: thrombotic microangiopathy after hematopoietic stem cell transplantation. Biol Blood Marrow Transplant 2005; 11:571-5.

2 Laskin BL, Goebel J, Davies SM, et al. Small vessels, big trouble in the kidneys and beyond: hematopoietic stem cell transplantation-associated thrombotic microangiopathy. Blood 2011;118:1452-62.

3 Radhi M, Carpenter SL. Thrombotic microangiopathies. Hematology 2012. http:/l www.isrn.com/journals/hematology/2012/310596/ (accessed 10 October 2012).

4 Myers JN, Shabshab SF, Burton NA, et al. Successful use of cyclosporine in a lung transplant recipient with tacrolimus-associated hemolytic uremic syndrome. J Heart Lung Transplant 1999;18:1024-6.

5 Hochberg MC. Updating the American College of Rheumatology revised criteria for the classification of systemic lupus erythematosus. Arthritis Rheum 1997;40:1725.

6 Wade $H$, Mori Y, Shimura M, et al . Poor outcome in disseminated intravascular coagulation or thrombotic thrombocytopenic purpura patients with severe vascular endothelial cell injuries. Am J Hematol 1998;58:189.

7 Trimarchi HM, Truong LD, Brennan S, et al. FK506-associated thrombotic microangiopathy: report of two cases and review of the literature. Transplantation 1999;67:539-44.

8 Romero L, Shah S, Kaza V. Tacrolimus induced hemolytic uremic syndrome in a lung transplant patient. Am J Respir Crit Care Med 2010;181:A5834.

9 Chhajed PN, Tamm M, Glanville AR. Role of flexible bronchoscopy in lung transplantation. Semin Resp and Crit Care Med 2004;25:31-43.

10 Fishman JA. Infection in solid-organ transplant recipients. N Engl J Med 2007;357:2601-14. 
Copyright 2013 BMJ Publishing Group. All rights reserved. For permission to reuse any of this content visit http://group.bmj.com/group/rights-licensing/permissions.

BMJ Case Report Fellows may re-use this article for personal use and teaching without any further permission.

Become a Fellow of BMJ Case Reports today and you can:

- Submit as many cases as you like

- Enjoy fast sympathetic peer review and rapid publication of accepted articles

- Access all the published articles

- Re-use any of the published material for personal use and teaching without further permission

For information on Institutional Fellowships contact consortiasales@bmjgroup.com

Visit casereports.bmj.com for more articles like this and to become a Fellow 